\title{
Experimental study on performance of contra-rotating axial flow fan
}

\author{
Shizhai Zhang ${ }^{1}$
}

Received: 2 February 2015/Revised: 2 June 2015 / Accepted: 8 June 2015/Published online: 30 July 2015

(C) The Author(s) 2015. This article is published with open access at Springerlink.com

\begin{abstract}
Contra-rotating axial flow fan is a kind of the vital equipment in coal mines. Their work conditions directly affect the safety of staff and production. In the paper, the performance of the contra-rotating axial flow fan is experimentally investigated. The study is focused on the fan performance, the shaft power and the match between the motor and fan efficiency at different blade angles. The results show that the blade angle $43^{\circ} / 26^{\circ}$ has the best aerodynamic performance. The first engine has a greater impact on the fan than the second one. The blade angle with the best aerodynamic performance does not necessarily correspond to the one with the best match between the motor and fan efficiency. The blade angle $43^{\circ} / 24^{\circ}$ is the best choice for the operation of the fan in the present study.
\end{abstract}

Keywords Contra-rotating axial flow fan $\cdot$ Fan performance $\cdot$ Shaft power $\cdot$ Blade angle

\section{List of symbols}

$\operatorname{Cos} \varphi \quad$ Power factor in Eq. (1)

$d \quad$ Diameter $(\mathrm{m})$

I Input current (A)

$K_{\mathrm{p}} \quad$ Correction factor

$N \quad$ Shaft power $(\mathrm{kW})$

$p \quad$ Pressure $(\mathrm{Pa})$

$\Delta p \quad$ Negative pressure of the collector $(\mathrm{Pa})$

$Q \quad$ Volumetric flow rate $\left(\mathrm{m}^{3} / \mathrm{min}\right)$

$t \quad$ Air temperature $\left({ }^{\circ} \mathrm{C}\right)$

$U \quad$ Input voltage (V)

\section{Greek symbols}

$\rho \quad$ Density $\left(\mathrm{kg} / \mathrm{m}^{3}\right)$

$\alpha_{n}$ Discharge coefficient of the collector in Eq. (2)

$\varepsilon_{n} \quad$ Expansion coefficient of the collector in Eq. (2)

$\eta \quad$ Efficiency

$\varphi \quad$ Atmosphere moisture in Eq. (3)

Shizhai Zhang

zhangshzh@126.com

1 Chongqing Research Institute, China Coal Technology Engineering Group, Chongqing 400039, China

\author{
Subscripts \\ a Atmosphere \\ el Electromotor \\ sat Saturated \\ st Static \\ sh Shaft \\ 1 Entrance state of the fan
}

\section{Introduction}

The main fan is the key equipment in the ventilation system of coal mine. Its role is to provide fresh air to the underground, and ensure the safety of staff and production. Also the main fan is the major power consumption equipment in coal mine because of its uninterrupted operation with long-term stability. Statistics indicated that the power consumption of the fan accounted for about $25 \%$ of the total amount in coal mine ( $\mathrm{Li}$ and Wang 2009). Therefore, it is of great importance for the safety and economic operation of the fan.

The contra-rotating axial flow fan is a fan whose two blades rotate in opposite direction. Figure 1 presents the construction of the contra-rotating axial flow fan. The fan mainly consists of seven parts, including collector, butterfly 


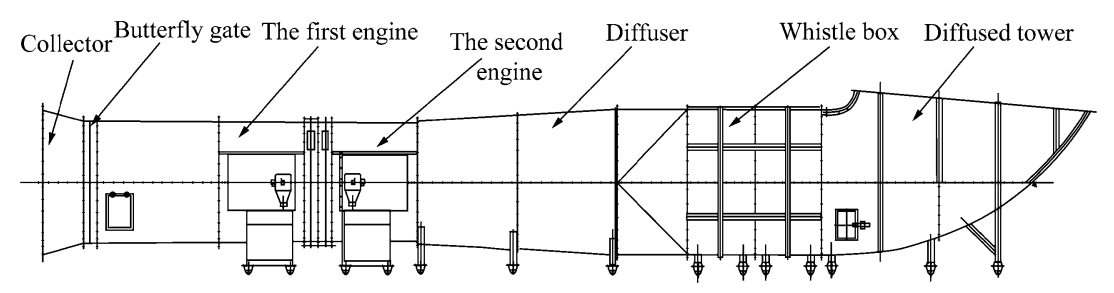

Fig. 1 Construction of the contra-rotating axial flow fan

gate, the first engine (impeller and motor), the second engine, diffuser, whistle box and diffused tower. During its working, the first impeller can be regarded as the front guide vane of the second one, likewise the second impeller can be the rear guide vane of the first one. Compared with the conventional fans, the contra-rotating axial flow fan raises the pressure ratio of the inlet to outlet within the limited space, thus improving the aerodynamic performance.

Investigations on the fans have been ongoing for the past decades. These works can be found in some popular volumes (Lakshminarayana 1996; McKenzie 1997; Bleier 1998). In recent years, a large number of studies have adopted numerical approaches, and these studies mostly focus on the modeling of the fan flow (Hung et al. 1999; Yu et al. 2005; Huang and Hsieh 2009; Gurina 2010; Hurault et al. 2010) and influence of tip flows on the fan performance (Jiang et al. 2001; Lee et al. 2003; Zhu et al. 2005) and noise generation (Jang et al. 2003; Jiang et al. 2007). However, few works were reported for the study of the contra-rotating axial flow fan. In the present article, we study the fan performance, the shaft power, and the match between the motor and fan efficiency at different blade angles.

\section{Experimental apparatus and data reduction}

\subsection{Experimental apparatus}

There are three ways to test the performance of the fan, including intake experiment, exhaust experiment, and intake and exhaust joint experiment. In the paper, we adopt intake experiment, as shown in Fig. 2. The air is inhaled into the pipe through the collector and distribution plate, then discharged from the fan. The fan performance is studied by adjusting the flow area of the pipe. The first pressure tap in Fig. 2 is used to measure the negative pressure of the collector, and the second pressure tap to measure the entrance static pressure of the fan.

In the experiment, the structural parameters of the contra-rotating axial flow fan are defined that the diameter is $2.4 \mathrm{~m}$, the hub ratio is 0.54 , the first impeller has 17 blades, and the second one has 15 blades. The volumetric flow rate of the fan ranges from 4200 to $8400 \mathrm{~m}^{3} / \mathrm{min}$, pressure ranging from 500 to $3100 \mathrm{~Pa}$. The nominal power of both motors is $185 \mathrm{~kW}$, and the rotating speed is $740 \mathrm{rpm}$. The test method is according to GB/T 1236-2000 "Industrial fans-Performance testing using standardized airways".

\subsection{Data reduction}

The test parameters mainly include the negative pressure of the collector, the entrance static pressure, the input current and voltage of both motors, atmosphere pressure and moisture, and air temperature.

The shaft power of the motor is calculated as

$N_{\text {sh }}=\frac{\sqrt{3} I U \cos \varphi}{1000} \eta_{\mathrm{el}}$

where $\cos \varphi$ is the power factor, and $\eta_{\mathrm{el}}$ is the electromotor transmission efficiency. The subscript 'sh' stands for the shaft.

The volumetric flow rate is measured with the collector, and calculated as (Zhou 2009)

$Q=\frac{\rho_{\mathrm{a}}}{\rho_{1}} Q_{\mathrm{a}}=\frac{66.643 \alpha_{n} \varepsilon_{n} d_{n}^{2} \sqrt{\rho_{\mathrm{a}} \Delta p_{n}}}{\rho_{1}}$

where $\alpha_{n}$ is the discharge coefficient of the collector, $\alpha_{n}=0.99$, and $\varepsilon_{n}$ is the expansion coefficient of the collector, $\varepsilon_{n}=1.0 . \Delta p_{n}$ is the negative pressure of the collector. The subscripts 'a' and ' 1 ' stand for the atmospheric

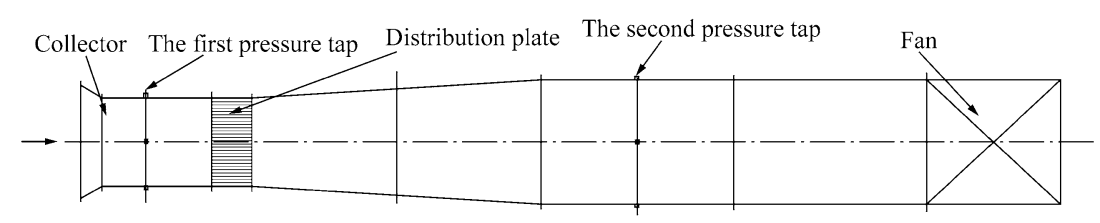

Fig. 2 The sketch of the experimental apparatus 
state and entrance state of the fan, respectively. The density at atmospheric state $\rho_{\mathrm{a}}$ is calculated as

$\rho_{\mathrm{a}}=3.484 \times 10^{-3} \frac{p_{\mathrm{a}}-0.3779 \varphi p_{\text {sat }}}{273+t}$

where $p_{\mathrm{a}}$ is the atmosphere pressure, $\varphi$ is the atmosphere moisture, and $p_{\text {sat }}$ is the saturated vapor pressure at the air temperature $t$. The density at entrance state $\rho_{1}$ is calculated as

$\rho_{1}=\rho_{\mathrm{a}} \frac{p_{1}}{p_{\mathrm{a}}}$

The static pressure efficiency is calculated as

$\eta_{\mathrm{st}}=\frac{Q p_{\mathrm{st}} K_{\mathrm{p}, \mathrm{st}}}{6.0 \times 10^{4} N_{\mathrm{sh}}}$

where $K_{\mathrm{p}}$ is the correction factor of the motor. The subscript 'st' stands for static.

\section{Results and discussion}

\subsection{Fan performance}

Experimental study on the performance of the contra-rotating axial flow fan is conducted at different blade angles. Three types of blade angles, $43^{\circ} / 24^{\circ}$ (the first and second blade angle), $43^{\circ} / 26^{\circ}$ and $40^{\circ} / 26^{\circ}$ are adopted in the paper.

Generally, the static pressure and the flow rate are mostly used as indices to characterize the fan performance. The relationship between the static pressure and the fan flow rate is usually referred as the performance curve, as shown in Fig. 3. It can be seen that the static pressure decreases with the increase of volumetric flow rate. When the first blade angle is $43^{\circ}$, the static pressure increases with the increase of the second blade angle from $24^{\circ}$ to $26^{\circ}$. When the second blade angle is $26^{\circ}$, the static pressure also increases with the increase of the first blade angle from $40^{\circ}$ to $43^{\circ}$. These phenomena are also found in the static pressure efficiency in Fig. 4. The dotted lines in Fig. 4

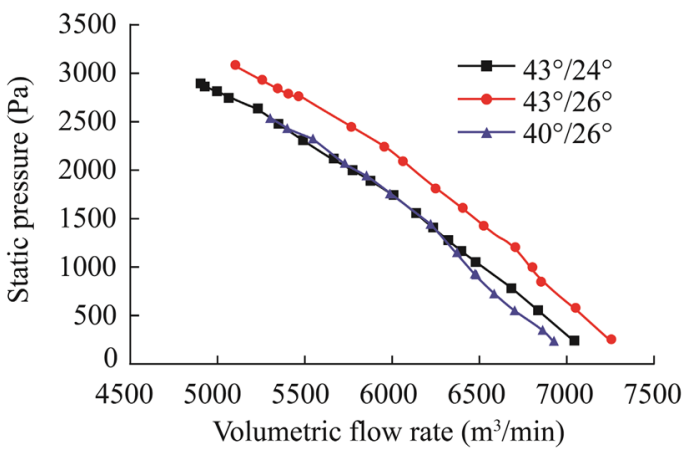

Fig. 3 Relationship between the static pressure and the fan volumetric flow rate

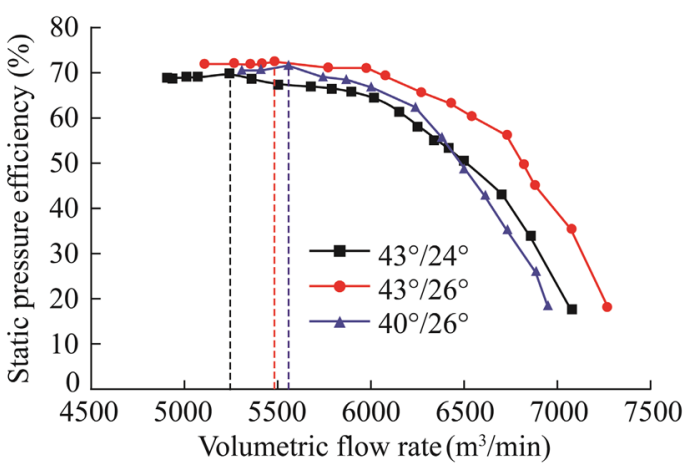

Fig. 4 Relationship between the static pressure efficiency and the fan volumetric flow rate

represent their highest efficiency point. We can conclude from Figs. 3 and 4 that the blade angle $43^{\circ} / 26^{\circ}$ has the best aerodynamic performance. The blade angle $40^{\circ} / 26^{\circ}$ is the second and the blade angle $43^{\circ} / 24^{\circ}$ is the last.

\subsection{Shaft power}

As mentioned above, the fan is one kind of the major energy consumption equipment in coal mines. In order to run the fan economically, the shaft power of the two motors is studied with different blade angles in this section.

Figure $5 \mathrm{a}, \mathrm{b}$ illustrates the shaft power of both motors at $43^{\circ} / 24^{\circ}$ and $43^{\circ} / 26^{\circ}$. The shaft power decreases with the increase of volumetric flow rate. This curve is similar to that of the fan performance in Fig. 3. It can be seen from

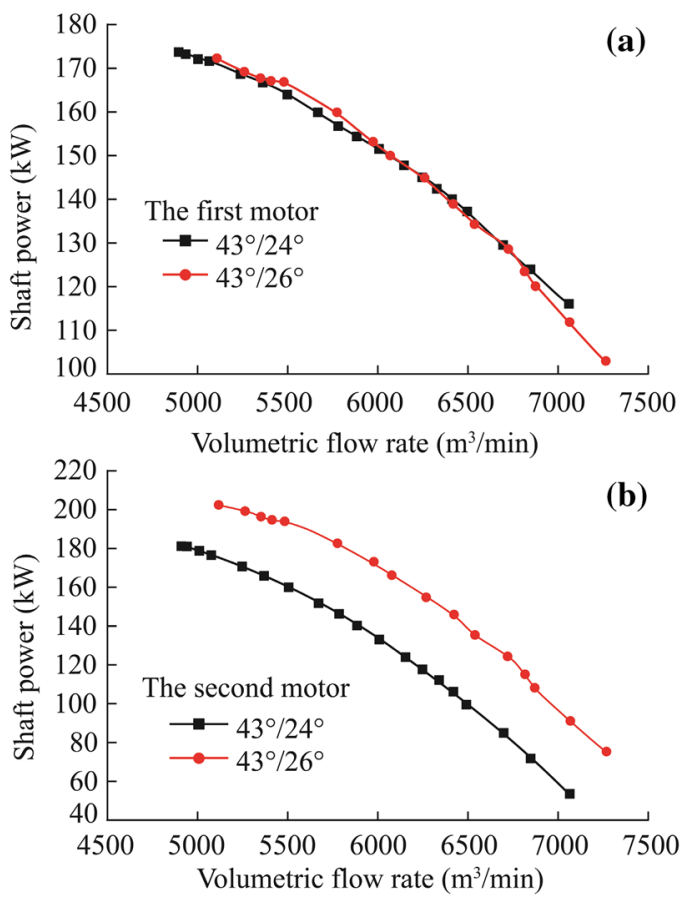

Fig. 5 The shaft power of both motors at $43^{\circ} / 24^{\circ}$ and $43^{\circ} / 26^{\circ}$ 
Fig. 5a that when the first blade remains the same angle $43^{\circ}$, the shaft power of the first motor coincides with each other. The variations of the second blade angle do not affect the shaft power of the first engine. This may be explained that when the first blade angle remains the same, the performance curve of the first engine does not change. The second engine can not affect the upstream flow. Consequently, the first motor consumption remains the same. With the increase of the second blade angle from $24^{\circ}$ to $26^{\circ}$ in Fig. 5 b, the shaft power of the second motor also increases. This is due to the change of the performance curve of the second engine.

Figure $6 \mathrm{a}, \mathrm{b}$ illustrates the shaft power of both motors at $43^{\circ} / 26^{\circ}$ and $40^{\circ} / 26^{\circ}$. It can be seen that when the first blade angle increases from $40^{\circ}$ to $43^{\circ}$ in Fig. $6 \mathrm{a}$, the shaft power of the first motor increases. The reason is the change of the performance curve of the first engine. However, although the second blades remain the same angle $26^{\circ}$, the shaft power of the second motor slightly increases in Fig. $6 \mathrm{~b}$. Also this is caused by the change of the first blade angle. The first impeller can be regarded as the front guide vane of the second one, which leads to the change of inlet velocity of the second impeller, thus altering the shaft power.

\subsection{The match between the motor and fan efficiency}

It is of vital importance for the continuous and steady operation of the fan in coal mine. The match between the motor and fan efficiency is directly related to the work
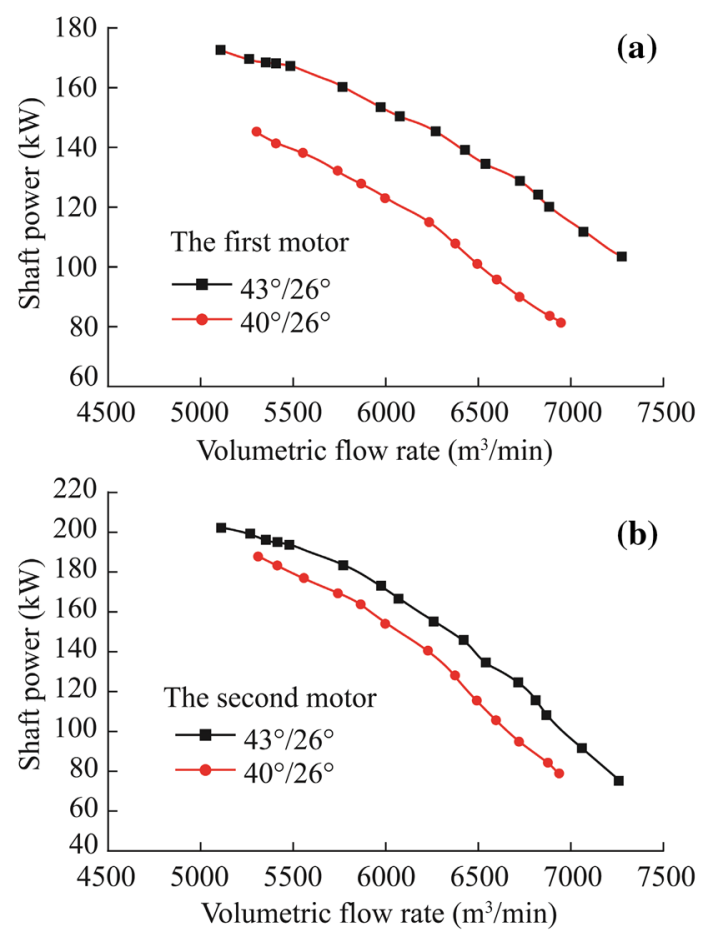

Fig. 6 The shaft power of both motors at $43^{\circ} / 26^{\circ}$ and $40^{\circ} / 26^{\circ}$ condition of the fan. In this section, the match relation is discussed at different blade angles.

Figure $7 \mathrm{a}-\mathrm{c}$ shows the shaft power of both motors at different blade angles. It can be seen that the gradient of the first motor shaft power is larger than that of the second motor shaft power, which indicates that the first engine has a greater impact on the fan than the second one. The dotted line in each figure represents the highest efficiency point. In Fig. $7 \mathrm{a}$, the shaft power of both motors is almost the same in the highest efficiency point at $43^{\circ} / 24^{\circ}$. The shaft power difference of both motors in Fig. $7 \mathrm{~b}$ is $27 \mathrm{~kW}$ in the highest efficiency point at $43^{\circ} / 26^{\circ}$, and it is $40 \mathrm{~kW}$ at $43^{\circ} \%$ $26^{\circ}$ in Fig. 7c. The results show that the blade angle $43^{\circ} \%$ $24^{\circ}$ is the best match between the motor and fan efficiency. The blade angle $43^{\circ} / 26^{\circ}$ is the second and the blade angle $40^{\circ} / 26^{\circ}$ is the last. On the other hand, the blade angle with the best aerodynamic performance does not necessarily correspond to the one with the best match between the
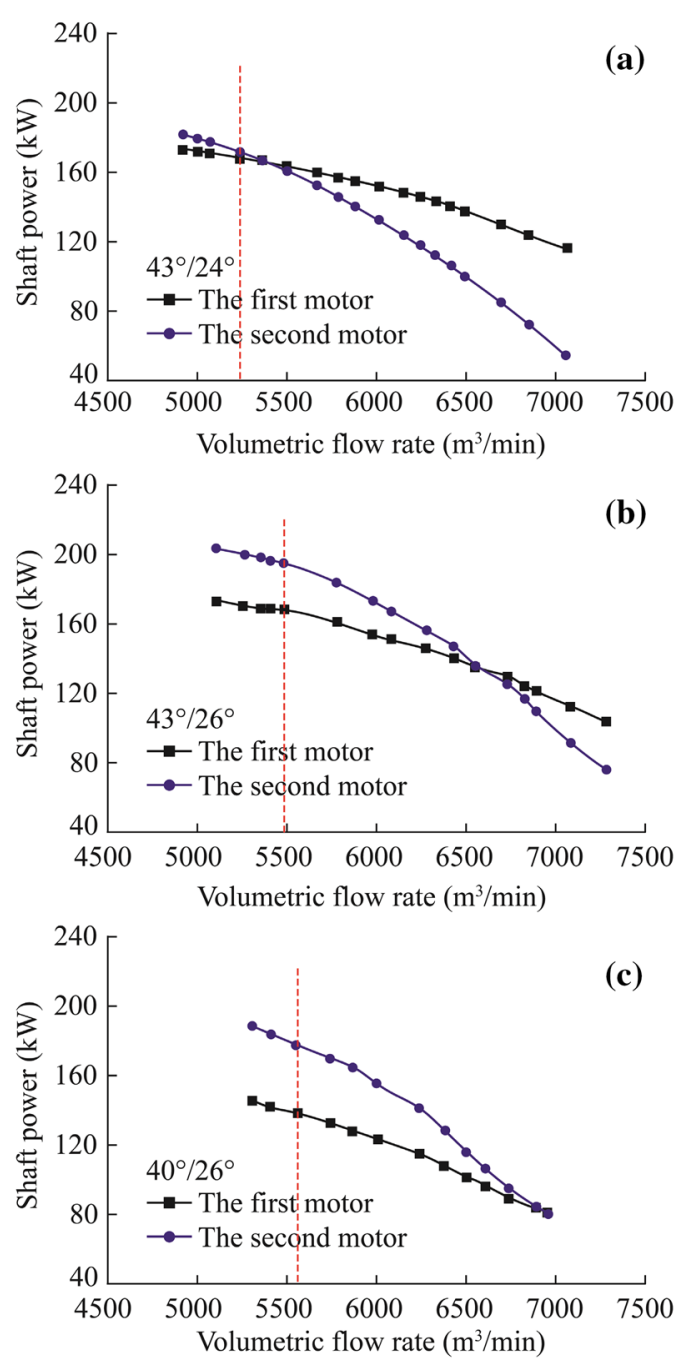

Fig. 7 The shaft power of both motors at different blade angles 
motor and fan efficiency. To ensure the safety production, the blade angle $43^{\circ} / 24^{\circ}$ is the best choice for the operation of the fan in the present study.

\section{Conclusions}

Experimental study on the performance of the contra-rotating axial flow fan has been presented in this paper. The following conclusions could be reached:

(1) The blade angle $43^{\circ} / 26^{\circ}$ has the best aerodynamic performance. The blade angle $40^{\circ} / 26^{\circ}$ is the second and the blade angle $43^{\circ} / 24^{\circ}$ is the last.

(2) When the first blade remains the same angle, the variations of the second blade angle do not affect the shaft power of the first engine. When the first blade angle increases and the second blade remain the same angle, the shaft power of both motors increases.

(3) The first engine has a greater impact on the fan than the second one. The blade angle with the best aerodynamic performance does not necessarily correspond to the one with the best match between the motor and fan efficiency. The blade angle $43^{\circ} / 24^{\circ}$ is the best choice for the operation of the fan in the present study.

Open Access This article is distributed under the terms of the Creative Commons Attribution 4.0 International License (http://crea tivecommons.org/licenses/by/4.0/), which permits unrestricted use, distribution, and reproduction in any medium, provided you give appropriate credit to the original author(s) and the source, provide a link to the Creative Commons license, and indicate if changes were made.

\section{References}

Bleier FP (1998) Fan handbook: selection, application, and design. McGraw-Hill, New York
Gurina EI (2010) Modeling of the operation of a mine counter rotation fan by means of the fluent suite. J Eng Phys Thermophys 83(5):985-990

Huang C, Hsieh M (2009) Performance analysis and optimized design of backward-curved airfoil centrifugal blowers. HVAC \& R Res 15(3):461-488

Hung C, Lam H, Dunn A (1999) Dynamic performance of an electronic zone air temperature control loop in a typical variable-air-volume air conditioning system. HVAC \& R Res 5(4):317-337

Hurault J, Kouidri S, Bakir F, Rey R (2010) Experimental and numerical study of the sweep effect on three-dimensional flow downstream of axial flow fans. Flow Meas Instrum 21(2):155-165

Jang C, Fukano M, Furukawa M (2003) Effects of the tip clearance on vertical flow and its relation to noise in an axial flow fan. JSME Int J Ser B 46(3):356-365

Jiang C, Furukawa M, Inoue M (2001) Analysis of vortical flow field in a propeller fan by LDV measurements and LES-part I: threedimensional vertical flow structures. ASME J Fluids Eng 123(4):748-754

Jiang C, Chen J, Chen Z, Tian J, Ouyang J, Du Z (2007) Experimental and numerical study on aeroacoustic sound of axial flow fan in room air conditioner. Appl Acoust 68(4):458-472

Lakshminarayana B (1996) Fluid dynamics and heat transfer of turbomachinery. Wiley, New York

Lee GH, Baek JH, Myung HJ (2003) Structure of tip leakage flow in a forward-swept axial-flow fan. Flow Turbul Combust 70:241-265

Li M, Wang X (2009) Performance evaluation methods and instrumentation for mine ventilation fans. Mining Sci Technol 19(6): 0819-0823

McKenzie AB (1997) Axial flow fans and compressors: aerodynamic design and performance. Ashgate, Aldershot

Yu Z, Li Z, He W, Wang W, Huang D, Zhu Z (2005) Numerical simulation of flow field for a whole centrifugal fan and analysis of the effects of blade inlet angle and impeller gap. HVAC \& R Res 11(2):263-283

Zhou H (2009) Design and research on testing system of the fan performance. PhD Dissertation. Chinese Academy of Agricultural Mechanization Sciences

Zhu X, Lin W, Du Z (2005) Experimental and numerical investigation on the flow field in the tip region of an axial ventilation fan. ASME J Fluids Eng 127(2):299-307 